\title{
PhysIOpathology of NEuromuscular function rElated to fatigue in chronic Renal disease in the elderly (PIONEER): study protocol
}

Antoine Chatrenet ${ }^{1,2^{*}}$ (D) Bruno Beaune ${ }^{2 \dagger}$, Antioco Fois ${ }^{1 \dagger}$, Camille Pouliquen ${ }^{2 \dagger}$, Jean-Michel Audebrand ${ }^{3}$, Massimo Torreggiani $i^{1}$, Damien Paris ${ }^{1,2}$, Sylvain Durand ${ }^{2+}$ and Giorgina Barbara Piccoli ${ }^{1,4+}$

\begin{abstract}
Background: Chronic Kidney Disease (CKD) is associated with reduced muscular strength resulting in profound fatigue. The physiopathology of these changes, their prevalence and evolution are still debated. Moreover, we have little data on elderly CKD patients. The present study protocol aims to 1) quantify the prevalence of low muscle strength (dynapenia) in a cohort of elderly patients with advanced CKD and to 2) characterize their force production coupled with electromyographic features and the symptoms of fatigue compared to a matched control group.

Methods: This is a case-control, prospective, interventional study. Inclusion criteria: age $\geq 60$ years; CKD Stage 3b-5; clinical stability (i.e. no hospitalization and $\leq 25 \%$ in creatinine increase in the previous 3 months). Controls with normal kidney function will be matched in terms of age, gender and diabetes mellitus (requisite: estimated glomerular filtration rate $\geq 60 \mathrm{ml} / \mathrm{min} / 1.73 \mathrm{~m}^{2}$ available in the last 6 months). Exclusion criteria for cases and controls: neuromuscular disease, life expectancy $<3$ months.

The handgrip strength protocol is an intermittent test consisting in 6 series of 9 repetitions of 3-s sub-maximum contractions at $40 \%$ of the maximum voluntary contraction (MVC) and $2 \mathrm{~s}$ of resting time between contractions. Each series is separated by one fast sub-maximum contraction and one MVC. Strength is assessed with a highfrequency handgrip dynamometer paired with surface electromyography. Symptoms of fatigue are assessed using MFI-20 and FACIT-F questionnaires. In order to reach a statistical power of 96\%, we plan to enroll 110 subjects in each group.

(Continued on next page)
\end{abstract}

\footnotetext{
* Correspondence: antoine.chatrenet@gmail.com

${ }^{\dagger}$ Bruno Beaune, Antioco Fois, Camille Pouliquen, Sylvain Durand and

Giorgina Barbara Piccoli contributed equally to this work.

'Nephrology, Centre Hospitalier Le Mans, Le Mans, France

¿Laboratory "Movement, Interactions, Performance" (EA 4334), Le Mans

University, Le Mans, France

Full list of author information is available at the end of the article
}

C C The Author(s). 2020 Open Access This article is licensed under a Creative Commons Attribution 4.0 International License, which permits use, sharing, adaptation, distribution and reproduction in any medium or format, as long as you give appropriate credit to the original author(s) and the source, provide a link to the Creative Commons licence, and indicate if changes were made. The images or other third party material in this article are included in the article's Creative Commons licence, unless indicated otherwise in a credit line to the material. If material is not included in the article's Creative Commons licence and your intended use is not permitted by statutory regulation or exceeds the permitted use, you will need to obtain permission directly from the copyright holder. To view a copy of this licence, visit http://creativecommons.org/licenses/by/4.0/ The Creative Commons Public Domain Dedication waiver (http://creativecommons.org/publicdomain/zero/1.0/) applies to the data made available in this article, unless otherwise stated in a credit line to the data. 


\begin{abstract}
(Continued from previous page)
Discussion: The novelty of this study resides in the application of an already validated set of tests in a population in which this combination (dynamometer, electromyography and questionnaires) has not previously been explored. We expect a high prevalence of dynapenia and a higher fatigability in CKD patients. A positive correlation is expected between reported fatigue and fatigability. Better appreciation of the prevalence and the relationship between fatigability and a sensation of fatigue can help us target interventions in CKD patients to improve quality of life and survival.
\end{abstract}

Trial registration: The study was approved by Ethical Committee EST III n²0.03.01 and was recorded as a Clinical Trial (NCT04330807) on April 2, 2020.

Keywords: Muscle fatigue, Tiredness, Electromyographic features, Pre-dialysis

\section{Background}

Chronic kidney disease (CKD) is defined as a structural or functional abnormality of the kidneys that has lasted at least 3 months and has implications for health [1]. The kidneys have a major role in the maintenance of homeostasis, ensuring water and electrolyte balance that directly determines the functioning of vital organs. It is for this reason that a decline in kidney function induces a number of systemic metabolic alterations, especially in the most advanced stages i.e., glomerular filtration rate (GFR): $\leq 45 \mathrm{ml} / \mathrm{min} / 1.73 \mathrm{~m}^{2}$ [2].

Among the symptoms associated with advanced CKD, fatigue is often reported by patients, with a prevalence ranging between 50 and $70 \%$ [3]. Fatigue is defined as a subjective sensation of weakness, increasing sense of effort, mismatch between exerted and actual effort [4]. Fatigue, which has a remarkable impact on a patient's everyday activities and quality of life, can be subjectively evaluated using validated questionnaires.

The pathogenesis of fatigue is complex. Fatigue can arise from disease-related issues (e.g., diffuse vascular disease, anemia, changes in lifestyle habits), psychological conditions (e.g., stress, depression, sleep disorders) and pathophysiological changes (e.g., decline in aerobic capacity or muscle strength) [5]. These problems are common in other chronic diseases, such as the consequences of stroke [6], cancer $[7,8]$, multiple sclerosis [9], rheumatoid arthritis [10,11] and diabetes mellitus [12-15]. In all of them, observed neuromuscular changes are associated with fatigue [16].

Neuromuscular fatigability is defined as a reduction in the patient's muscular ability to perform a standardized task [17]. In general, inability to exert the desired force is not restricted to muscle-mass decline. Several factors concur to determine muscle function: fiber type (i.e., slow or fast twitches), metabolism (i.e., aerobic or anaerobic), presence of fat infiltration or fibrosis and interactions at the neuromuscular junction [18]. CKD induces chronic muscle function changes [19-21]: this is relatively well documented for dialysis patients [22, 23], but we still have little data about the loss of muscle strength (dynapenia [24]) in CKD patients.

In the course of chronic dialysis, the loss of muscle function is worsened by several factors, such as acidosis, loss of amino acids (up to $12 \mathrm{~g}$ per hemodialysis session or 4 to $6 \mathrm{~g}$ per day on peritoneal dialysis) and/or the presence of anorexia. In this context, the loss of strength is mainly attributable to a decrease in muscle-mass, characterized by the loss, or a decrease in size, of the contractile units [2527]. Conversely, changes in the excitationcontraction coupling system have been little explored and are poorly understood, especially in patients with advanced CKD.

The grip isometric analysis (i.e., handgrip) is the most commonly used test to assess strength in patients who have advanced CKD [28]. The handgrip test is simple and rapidly performed; it yields reproducible results and can easily be integrated into routine clinical practice. In CKD patients, this test demonstrates a close correlation with survival, nutritional status and quality of life [29-33]. Moreover, an isometric contraction is less traumatic than a dynamic contraction [34]. Finally, a handgrip is a simple routine gesture and does not usually cause pain.

Thus, the aim of the study is to assess the neuromuscular features of elderly patients with advanced CKD and symptoms of fatigue coupled with dynapenia. To our knowledge, this is the first study to apply a complex set of validated tests to a large population of elderly patients who have advanced CKD but are not on dialysis, with respect to an age, sex and diabetes matched control group. The research will highlight neuromuscular features associated with dynapenia in elderly CKD patients. In addition, the relationship between dynapenia and subjective fatigue will be analyzed to identify novel therapeutic interventions. These findings will be rapidly translated into clinical practice, with a view to adapting physical activity programs to improve neuromuscular function in the management of CKD symptoms. 


\section{Methods}

\section{Aims}

The primary objective of this trial is to define the prevalence of dynapenia in elderly CKD Stage 3b-5 patients. This will be achieved through the analysis of handgrip strength.

The secondary objective is to define the characteristics of dynapenia in elderly patients with advanced CKD during a standardized task considering:

a) the evolution of MVC;

b) the evolution of the discharge frequency of motor units;

c) motor-unit recruitment ability;

d) the evolution of the delay between the nervous stimulus and contraction;

e) the relationship between dynapenia and subjective fatigue.

\section{Clinical implications}

By shedding light on the specific characteristics of dynapenia and its link with a patient's sense of fatigue and fatigability [4], the PIONEER study intends to guide the management of adapted physical activities recommended by academic associations.

\section{Study design}

A case-control, interventional, prospective study.

\section{Setting of study}

The study will be carried out in Le Mans hospital (France), in the outpatient unit for the care of advanced kidney disease (UIRAV, Unité pour l'Insuffisance Rénale chronique AVancée). The UIRAV unit follows about 210 patients which are not on dialysis $(90 \%$ over the age of 60 ), whose mean age is 74 years; $60 \%$ are males and diabetes mellitus prevalence is approximately $50 \%$. Of these, 180 patients are followed for CKD Stage 3b-5. As previously described by Fois et al. [35], UIRAV's main objective is to offer frequent follow-up and to delay the start of dialysis. The frequency of controls is based on CKD stage and overall clinical stability. For example, patients with stable kidney disease Stage $3 \mathrm{~b}$ are seen every 8 to 12 weeks, while patients with CKD Stage 5 are seen at least once a month. Study proceedings is described in Fig. 1.

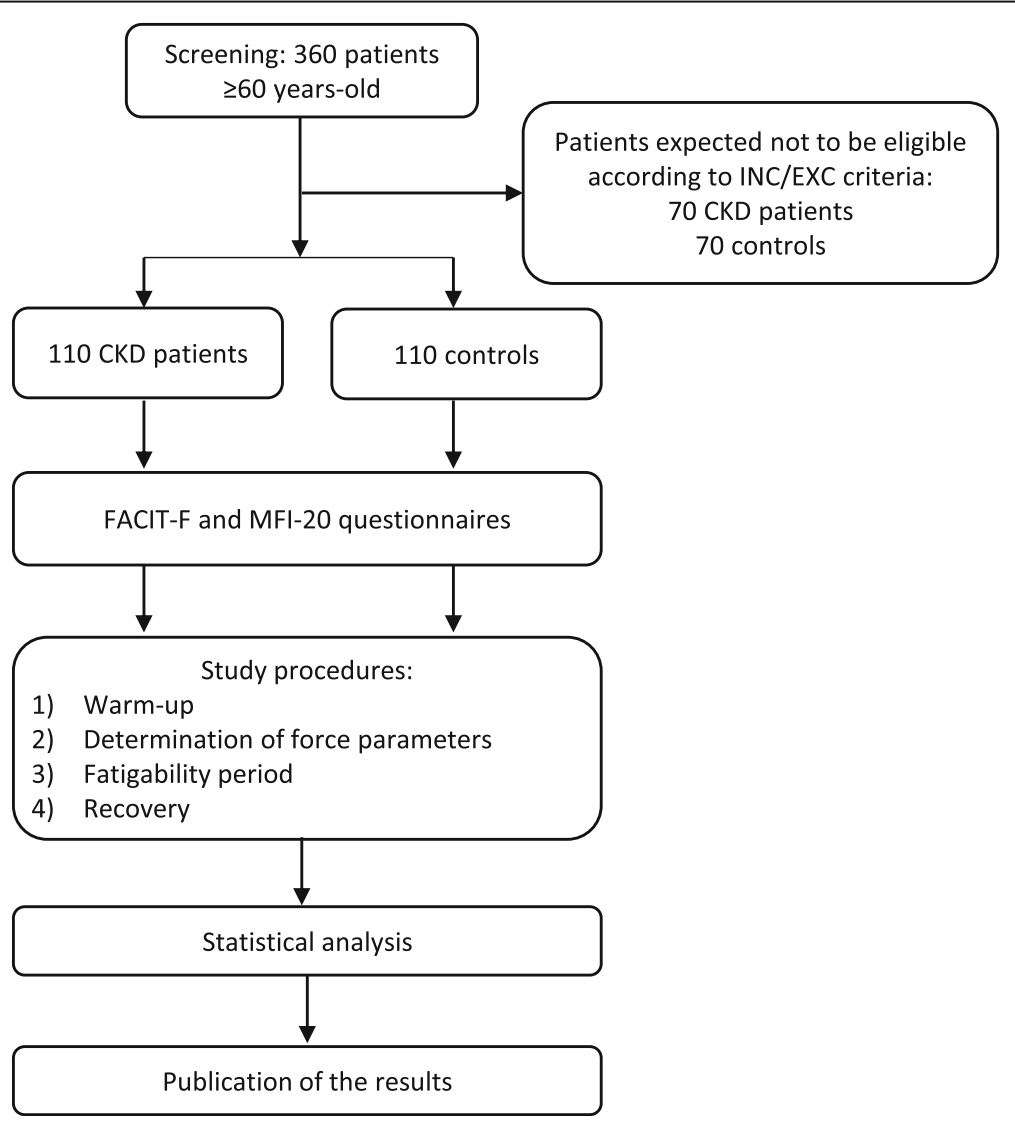

Fig. 1 Flowchart of the study. INC: inclusion criteria; EXC: exclusion criteria 


\section{Definition and measures \\ Dynapenia}

Dynapenia is an age-associated loss of muscle strength that is not caused by neurologic or muscle diseases [24]. In our study, dynapenia will be diagnosed if the mean of three MVC after a brief warm up, is lower than the reference value matched for age and sex. The study by Ramírez-Vélez et al. [36] will provide reference values for elderly patients.

\section{Fatigability}

Fatigability is defined as a reduction in the patient's muscle performance on a standardized task [17]. Fatigability, which is an indicator of objective fatigue, is evaluated by measuring critical force $\left(\mathrm{F}_{\text {crit }}\right)$ during a standardized task. The $\mathrm{F}_{\text {crit }}$ value corresponds to the asymptotic value of the last stable part of the MVC evolution curve (Fig. 2) [37]. $F_{\text {crit }}$ is the maximum exercise intensity that a subject can produce in a given metabolic environment, in other words, it identifies the threshold at which fatigability develops [38].

\section{Fatigue}

The perception of fatigue is defined as a subjective sensation of weariness, increasing sense of effort, mismatch between expended and actual effort [4]. We will use two questionnaires to assess fatigue: the Functional Assessment of Chronic Illness Therapy-Fatigue (FACIT-F) and the Multidimensional Fatigue Inventory (MFI-20).

FACIT-F is a brief validated French-language questionnaire [39] consisting in 13 simple pragmatic statements (e.g., I am too tired to eat) accompanied by the Likert scale (0: "never or almost never" to 4: "always or almost always"). The final score ranges from 0 to 52 , with an inverse relationship between the score and fatigue.

MFI-20 is also a validated French-language version of a widely-used questionnaire [40], focusing on fatigue. It is composed of statements covering self-perception, ranging from extremely positive (e.g., physically I feel/I am in excellent condition) to extremely negative (e.g., physically I feel/I am able to do very little). In the case of the MFI-20, there is a direct relationship between the patient's score and perceived fatigue while exploring 4 topics: general fatigue, mental fatigue, reduced activity and motivation.

\section{Anthropometric, clinical, nutritional and biochemical data}

Relevant data will be collected from medical records. They will include age, body mass index, gender, presence

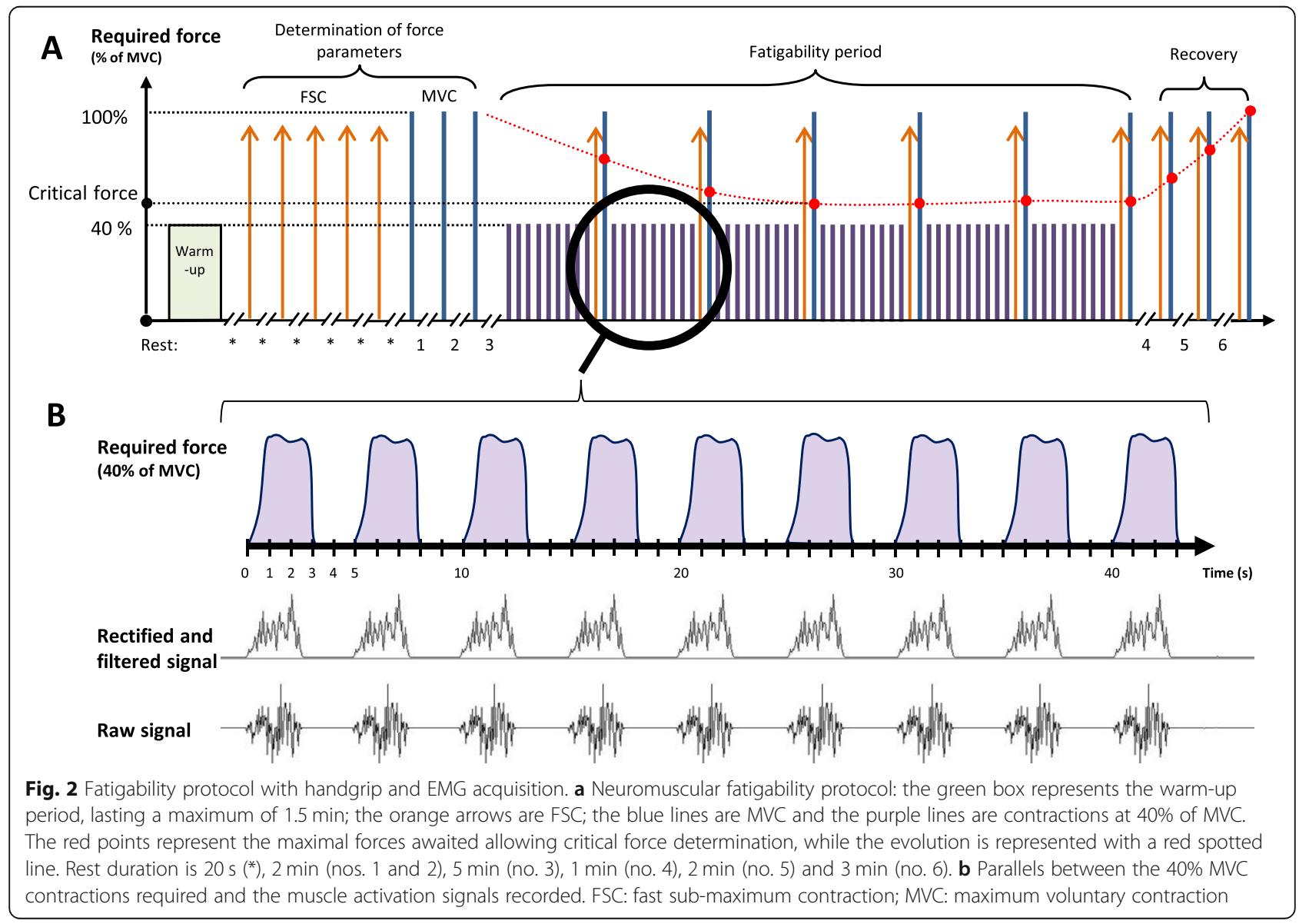


of diabetes mellitus (defined as glycemia $>126 \mathrm{mg} / \mathrm{dl}$ while fasting or using oral hypoglycemic medications or insulin) and kidney disease (i.e., cause and creatinine, urea and proteinuria values), time of follow-up, Charlson Comorbidity Index (CCI) [41], Malnutrition Inflammation Score (MIS) [42], albumin, total cholesterol, highdensity lipoprotein, low-density lipoprotein, triglycerides, uric acid, calcium, phosphorus, parathyroid-hormone, vitamin $\mathrm{D}$, bicarbonate, sodium and potassium values. The most recent available data will be recorded within 3 months for CKD patients and 6 months for control volunteers.

\section{Inclusion criteria \\ Cases}

- Age $\geq 60$

- Estimated glomerular filtration rate based on the Chronic Kidney Disease Epidemiology Collaboration (CKD-EPI) equation $\leq 45 \mathrm{ml} / \mathrm{min} / 1.73 \mathrm{~m}^{2}$ for at least 3 months

- Stable clinical condition: no hospitalization; serum creatinine increased by a maximum of $25 \%$ in the previous 3 months

\section{Controls}

- Age $\geq 60$

- Available blood tests within 6 months showing normal renal function (estimated GFR $>60 \mathrm{ml} / \mathrm{min} /$ $1.73 \mathrm{~m}^{2}$ ) and glycated hemoglobin values for diabetic controls

- Stable clinical condition: no hospitalization in the previous 6 months

\section{Exclusion criteria}

- Inability to give informed consent, if under guardianship or a minor

- Neuromuscular disease

- Dementia

- History of upper limb surgery or pathologies that would make it impossible to fit electromyography (EMG) electrodes and measure handgrip force

- Estimated life expectancy of less than 3 months

- Hospitalization in the 3 months prior to the test

- Participation in another clinical interventional trial

- Acute kidney disease

- Waitlisted for renal replacement therapy or expected to start dialysis within 3 months

- History or evidence of any other clinically significant disorder, condition or disease that in the investigator's opinion could pose a risk to a participant's safety or interfere with the study's evaluation, procedures or completion

\section{Inclusion modalities}

Participants will be informed about the study with a poster displayed in hospital or during medical consultations. Elderly CKD patients will be enrolled via the UIRAV unit. Control subjects will be enrolled through endocrinology, diabetology and dermatology units or during outpatient consultations with patients' escorts.

\section{Materials}

The handgrip and EMG evaluations will be synchronized using Labview v19 software (National Instruments Corp., Austin TX, USA) that makes it possible to simultaneously display feedback on strength and EMG activity. The handgrip dynamometer used will be the K-Force GRIP (K-Invent Inc., Montpellier, France), with $1000 \mathrm{~Hz}$ acquisition frequency and an accuracy of $100 \mathrm{~g}$. The EMG signal will be recorded using the Trigno Wireless Biofeedback System (DelSys Inc., Boston MA, USA) with the Trigno Avanti ${ }^{\mathrm{Tm}}$ electrodes, which are composed of a 4 silver bar contacts, $10 \mathrm{~mm}$ interelectrode, a $1926 \mathrm{~Hz}$ acquisition frequency per channel and with a secondorder band-pass Butterworth filter set at $20-450 \mathrm{~Hz}$ and a second-order low-pass filter set at $100 \mathrm{~Hz}$.

\section{Intervention}

The patient being tested will be seated, with his back in a straight position, and elbow bent at $90^{\circ}$ close to his chest. The patient's humerus will be vertical and his forearm will be parallel to a height-adjustable support (Fig. 3). The patient's dominant arm will be chosen.

Preparing the arm for placement of the electrodes will include shaving the skin and cleaning the area hosting the four recording electrodes $[43,44]$ with a sponge and alcoholic disinfectant to reduce impedance. Electrodes will be placed over the flexor digitorum superficialis muscle belly along the fiber direction while following international SENIAM recommendations and the four electrode positions previously described [43-45].

The exercise, illustrated in Fig. 2, will begin with a warm-up, to familiarize the person with the instruments and situation, at a slow and comfortable pace not exceeding $1 \mathrm{~min}$ and $30 \mathrm{sec}$. The warm-up will consist of dynamic extensions of the hand and fingers: an extension at maximum speed, and a slow and constant flexion of the wrist and fingers. Dynamic stretches have been shown to prepare the nervous system, increase muscle temperature and improve the sensitivity of the connecting bridges between actin and myosin [46] compared to passive stretches, which can be somewhat deleterious in terms of muscle performance [47]. 
Screen displaying:

- Force and EMG feedback

-Protocol indications

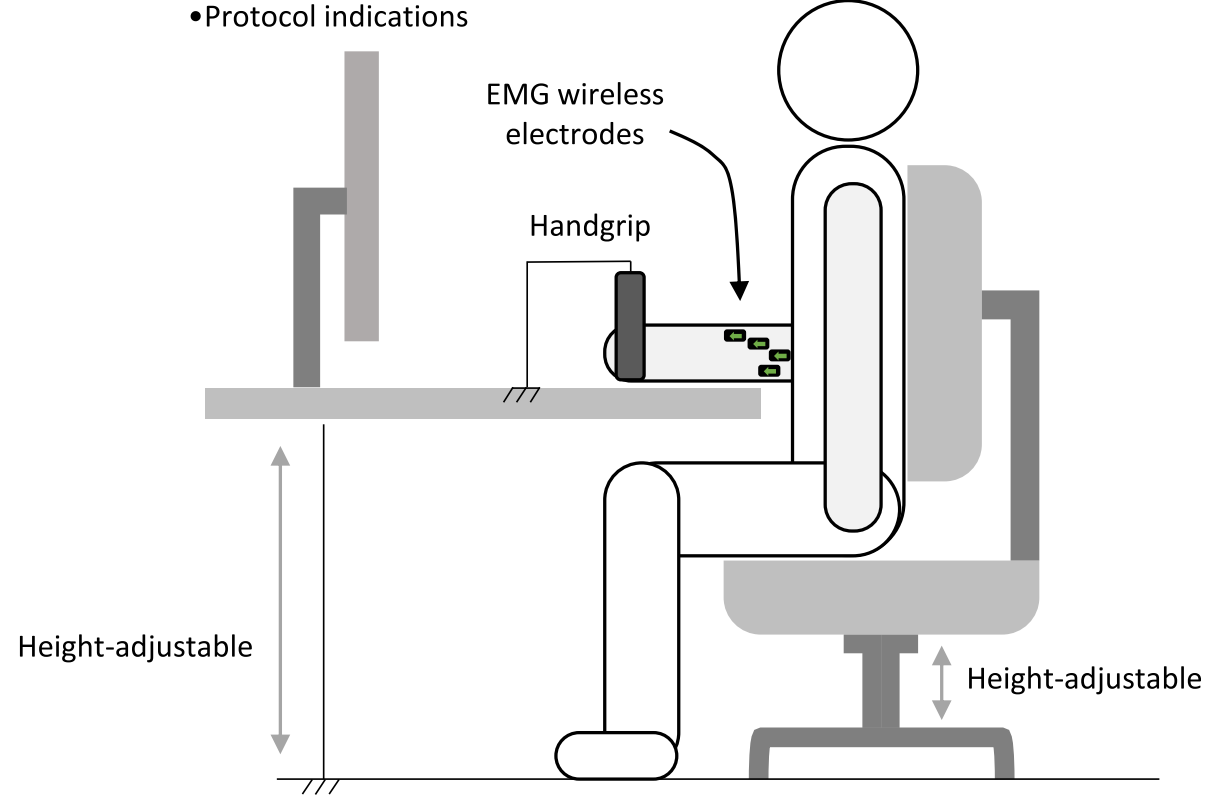

Fig. 3 Set-up of the study and standardized position

According to the recommendations of Maffiuletti et al. [48], fast sub-maximum contractions (FSC) should be separated from MVC to limit central inhibition. Thus, to determine the baseline FSC, five contractions at maximum speed will be performed with a twenty-second rest interval between them. The objective of these contractions is to determine the rate of force development.

As recommended by De Luca [49], patients will perform three MVC interspersed by a two-minute rest and the mean of the three contractions will be calculated. Each MVC has to be reached progressively in less than 3 sec and least $5 \mathrm{sec}$ [50]. A five-minute break is scheduled after the MVC because the subsequent part of the trial is the beginning of the fatigability protocol.

The fatigability assessment phase begins with nine contractions at $40 \%$ of MVC [51], each lasting $3 \mathrm{sec}$, followed by an FSC and an MVC. All the contractions are exerted at two-second intervals. This cycle of eleven contractions will be repeated six times. If a patient cannot maintain the $40 \%$ required force, the threshold time will be recorded without stopping the test. At the end of these cycles, the patient will rest for $1 \mathrm{~min}$. Then, in order to assess their recovery capacities, the last part of the test will be administered, consisting in three series of one FSC and one MVC, separated by two- and threeminute intervals, respectively.

\section{Statistical analysis}

Sample size calculation was based on the results of the handgrip strength test of Lin et al. [52], who analyzed a relatively large cohort of elderly patients suffering from CKD in 2019, and the handgrip strength reference values of Ramírez-Vélez et al. [36], who analyzed a larger population of elderly patients without CKD, stratified by age and sex. With an $8 \%( \pm 16 \%)$ difference, accepting a $5 \%$ alpha risk, $96 \%$ statistical power will be reached by enrolling $110 \mathrm{CKD}$ patients and 110 control subjects. Considering the number of patients followed in the UIRAV unit (i.e., approximately 180 older than 60), we should be able to enroll the participants within 1 year.

Signal filtering and analysis will be performed with Matlab 2018a v9.4 (The MathWorks Inc., Natick MA, USA) and statistical analysis will be performed with SPSS v14 (IBM Corp., Armonk NY, USA). Group comparison for normally distributed variables will be assessed by means of the analysis of variance (ANOVA) or the unpaired Student T-Test. For non-normally distributed variables the Kruskal-Wallis or the MannWhitney Test will be used. In addition, the chronological variables (e.g., evolution of the MVC during the test) will be analyzed by mixed models with fixed effect or ANOVA with repeated measures if the test prerequisites are satisfied (normality, homoscedasticity and sphericity).

Qualitative data will be compared with the $\mathrm{Chi}^{2}$ or the McNemar Test.

Correlation analyses will be performed with the Pearson Test. Logistic or multiple regressions will be performed to assess the effect of different variables on the outcomes: age, sex, body mass index, stage of renal 
disease, diabetes mellitus, MIS and CCI (either continuous or dichotomized) as well as biochemical data. A $p<$ 0.05 will be considered statistically significant.

\section{Discussion}

This study aims to quantify the prevalence of force decline in elderly patients affected by advanced CKD and to identify alterations at the level of the neuromuscular junction. The strength of this study is that it will be, to our knowledge, the first study to assess, in this "pre-dialysis" population, the prevalence of dynapenia coupled with an EMG recording to objectivate electromyographic fatigability features. It will also be the first trial combining an objective (i.e., $\mathrm{F}_{\text {crit }}$ ) and a subjective (i.e., FACIT-F and MFI-20) approach to assess fatigue in this population. The link between neuromuscular alterations and greater fatigue is in fact poorly understood [16], especially for CKD patients.

A higher prevalence of dynapenia is expected in the CKD population compared to the control group, with the highest prevalence in those with diabetes mellitus. Patients with diabetes appear to have decreased muscle functions [53, 54], but this result has been questioned [55]. We expect different neuromuscular features in CKD patients, specifically for $F_{\text {crit }}$. CKD has been associated with difficulty in performing a standardized task but the origin of this disturbance (e.g., difficulty adapting the motor-unit discharge or difficulty recruiting the motor unit) is far less clear [48]. Our study will allow us to investigate these issues and clarify the underlying pathophysiological mechanisms. The temporal difference between the onset of the EMG signal and force production, i.e. the electromechanical delay, will be assessed during the FSC. We expect an increase in this delay, highlighting an alteration between the electrochemical process (i.e., synaptic transmission, propagation of the action potential, excitation-contraction coupling) and contractile and structural elements. A positive correlation is expected between subjective fatigue and objective fatigability.

The frailty of the elderly CKD population demands careful attention to the methodology employed, explaining the choice of this sub-maximum protocol to reduce as far as possible causing pain while inducing sufficient fatigue in this fragile population.

Better comprehension of the neuromuscular features of dynapenia and a deeper understanding of the relationship between fatigability and fatigue can help us guide interventions for elderly patients suffering from advanced $C K D$, with the final goal of targeting interventions to effectively improve quality of life and reduce morbidity.

\section{Abbreviations}

ANOVA: ANalysis Of VAriance; CCl: Charlson Comorbidity Index; CKD: Chronic Kidney Disease; CKD-EPI: Chronic Kidney Disease EPIdemiology Collaboration; EMG: ElectroMyoGraphy; FACIT-F: Functional Assessment of Chronic Illness Therapy-Fatigue; Fcrit: critical Force; FSC: Fast Sub-maximum Contraction; GFR: Glomerular Filtration Rate; MFI: Multifactorial Fatigue Inventory; MIS: Malnutrition Inflammation Score; MVC: Maximum Voluntary Contraction; UIRAV: Unité pour l'Insuffisance Rénale AVancée

\section{Acknowledgments}

The authors would like to thank S. Finnel for careful language correction of the text and C. Jadeau, A. Le Cam and C. Perreto for ethical, organizational and administrative help.

\section{Authors' contributions}

AC designed the research protocol; AC and GBP defined inclusion/exclusion criteria for patients and controls and submitted the protocol to the Ethics Committee. AF was responsible for the description and adaptation of the organization; CP contributed to signal analysis and interpretation and JMA and DP carried out preliminary experiments; AC, MT, SD, BB and GBP wrote the text of this protocol, whose final draft was reviewed and approved by all the authors.

\section{Funding}

The PIONEER study is a PhD thesis-project conducted in the Le Mans Hospital and Le Mans University. The research project is promoted by the Le Mans Hospital and partially financed by the French Ministry of Training and Research via the National Association for Research and Technology by means of the Convention Industrielle de Formation par la Recherche (CIFRE) grant ( $\left.n^{\circ} 2018 / 1255\right)$. Half of the Ph.D. student's salary is paid through this grant and the other half by the hospital. The founding source has no role in the study design, data collection, results interpretation or manuscript writing.

\section{Availability of data and materials}

The details, location and accessibility of the results collected during this study will be reported in a peer-reviewed report, referencing this protocol article.

\section{Ethics approval and consent to participate}

The present study was approved by Ethical Committee Est-III of Nancy University Hospital Center $n^{\circ} 20.03 .01$, and was recorded (reference: ID-RCB: 2020-A00109-30) as a Clinical Trial (NCT: 04330807) on April 2, 2020. It will be conducted in accordance with the Declaration of Helsinki. All volunteers will receive an information letter, will have the possibility to discuss any questions they have with medical personnel and, if willing to participate, will sign an informed consent before being enrolled in the study. This protocol complies with the SPIRIT checklist.

\section{Consent for publication}

Not applicable.

\section{Competing interests}

The authors declare that there are no competing interests.

\section{Author details}

${ }^{1}$ Nephrology, Centre Hospitalier Le Mans, Le Mans, France. ${ }^{2}$ Laboratory "Movement, Interactions, Performance" (EA 4334), Le Mans University, Le Mans, France. ${ }^{3}$ Endocrinology and diabetology, Centre Hospitalier Le Mans, Le Mans, France. ${ }^{4}$ Department of clinical and biological sciences, University of Torino, Torino, Italy.

Received: 15 July 2020 Accepted: 22 July 2020

Published online: 25 July 2020

\section{References}

1. Levey AS, Eckardt K-U, Dorman NM, Christiansen SL, Hoorn EJ, Ingelfinger $J R$, et al. Nomenclature for kidney function and disease: report of a kidney disease: improving global outcomes (KDIGO) consensus conference. Kidney Int. 2020;97(6):1117-29.

2. Chmielewski M, Carrero JJ, Stenvinkel P, Lindholm B. Metabolic abnormalities in chronic kidney disease that contribute t cardiovascular 
disease, and nutritional initiatives that may diminish the risk. Curr Opin Lipidol. 2009;20:3-9.

3. Murtagh FEM, Addington-Hall J, Higginson IJ. The prevalence of symptoms in end-stage renal disease: a systematic review. Adv Chronic Kidney Dis. 2007;14:82-99.

4. Kluger BM, Krupp LB, Enoka RM. Fatigue and fatigability in neurologic illnesses: proposal for a unified taxonomy. Neurology. 2013;80:409-16.

5. Sawant A, Garland SJ, House AA, Overend TJ. Morphological, electrophysiological, and metabolic characteristics of skeletal muscle in people with end-stage renal disease: a critical review. Physiother Can. 2011; 63:355-76

6. Knorr S, Rice CL, Garland SJ. Perspective on neuromuscular factors in poststroke fatigue. Disabil Rehabil. 2012;34:2291-9.

7. Prinsen H, van Dijk JP, Zwarts MJ, Leer JWH, Bleijenberg G, van Laarhoven HWM. The role of central and peripheral muscle fatigue in postcancer fatigue: a randomized controlled trial. J Pain Symptom Manag. 2015;49:17382.

8. Yavuzsen T, Davis MP, Ranganathan VK, Walsh D, Siemionow V, Kirkova J, et al. Cancer-related fatigue: central or peripheral? J Pain Symptom Manag. 2009;38:587-96

9. Krupp LB, Serafin DJ, Christodoulou C. Multiple sclerosis-associated fatigue. Expert Rev Neurother. 2010;10:1437-47.

10. Staud R. Peripheral and central mechanisms of fatigue in inflammatory and noninflammatory rheumatic diseases. Curr Rheumatol Rep. 2012;14:539-48.

11. Wartolowska K, Hough MG, Jenkinson M, Andersson J, Wordsworth BP, Tracey I. Structural changes of the brain in rheumatoid arthritis. Arthritis Rheumatism. 2012;64:371-9.

12. Akashi PMH, Sacco ICN, Watari R, Hennig E. The effect of diabetic neuropathy and previous foot ulceration in EMG and ground reaction forces during gait. Clin Biomech (Bristol, Avon). 2008;23:584-92.

13. Allen MD, Major B, Kimpinski K, Doherty TJ, Rice CL. Skeletal muscle morphology and contractile function in relation to muscle denervation in diabetic neuropathy. J Appl Physiol. 2014;116:545-52.

14. Goedendorp MM, Tack CJ, Steggink E, Bloot L, Bazelmans E, Knoop H. Chronic fatigue in type 1 diabetes: highly prevalent but not explained by hyperglycemia or glucose variability. Diabetes Care. 2014;37:73-80.

15. Singh R, Kluding PM. Fatigue and related factors in people with type 2 diabetes. Diabetes Educ. 2013:39:320-6.

16. Twomey R, Aboodarda SJ, Kruger R, Culos-Reed SN, Temesi J, Millet GY. Neuromuscular fatigue during exercise: methodological considerations, etiology and potential role in chronic fatigue. Neurophysiol Clin. 2017;47: 95-110.

17. Bigland-Ritchie B, Woods JJ. Changes in muscle contractile properties and neural control during human muscular fatigue. Muscle Nerve. 1984;7:691-9.

18. McGregor RA, Cameron-Smith D, Poppitt SD. It is not just muscle mass: a review of muscle quality, composition and metabolism during ageing as determinants of muscle function and mobility in later life. Longev Healthspan. 2014;3:9

19. Hirai K, Ookawara S, Morishita Y. Sarcopenia and physical inactivity in patients with chronic kidney disease. Nephrourol Mon. 2016;8:e37443.

20. O'Sullivan TF, Smith AC, Watson EL. Satellite cell function, intramuscular inflammation and exercise in chronic kidney disease. Clin Kidney J. 2018;11: 810-21.

21. Wilkinson TJ, Gould DW, Nixon DGD, Watson EL, Smith AC. Quality over quantity? Association of skeletal muscle myosteatosis and myofibrosis on physical function in chronic kidney disease. Nephrol Dial Transplant. 2018; 34:1344-54.

22. Cheema B, Abas H, Smith B, O'Sullivan AJ, Chan M, Patwardhan A, et al. Investigation of skeletal muscle quantity and quality in end-stage renal disease. Nephrology (Carlton). 2010;15:454-63.

23. Fahal $I H$, Bell GM, Bone JM, Edwards RH. Physiological abnormalities of skeletal muscle in dialysis patients. Nephrol Dial Transplant. 1997;12:119-27.

24. Clark BC, Manini TM. What is dynapenia? Nutrition. 2012;28:495-503.

25. Fouque D, Kalantar-Zadeh K, Kopple J, Cano N, Chauveau P, Cuppari L, et al. A proposed nomenclature and diagnostic criteria for protein-energy wasting in acute and chronic kidney disease. Kidney Int. 2008;73:391-8.

26. Johansen KL, Kaysen GA, Young BS, Hung AM, da Silva M, Chertow GM. Longitudinal study of nutritional status, body composition, and physical function in hemodialysis patients. Am J Clin Nutr. 2003;77:842-6.

27. Raj DSC, Sun Y, Tzamaloukas AH. Hypercatabolism in dialysis patients. Curr Opin Nephrol Hypertens. 2008;17:589-94.
28. Leal VO, Mafra D, Fouque D, Anjos LA. Use of handgrip strength in the assessment of the muscle function of chronic kidney disease patients on dialysis: a systematic review. Nephrol Dial Transplant. 2011;26:1354-60.

29. Fried LF, Lee JS, Shlipak M, Chertow GM, Green C, Ding J, et al. Chronic kidney disease and functional limitation in older people: health, aging and body composition study. J Am Geriatr Soc. 2006;54:750-6.

30. Gracia-Iguacel C, González-Parra E, Pérez-Gómez MV, Mahíllo I, Egido J, Ortiz A, et al. Prevalence of protein-energy wasting syndrome and its association with mortality in haemodialysis patients in a Centre in Spain. Nefrologia. 2013:33:495-505.

31. Pagels AA, Söderkvist BK, Medin C, Hylander B, Heiwe S. Health-related quality of life in different stages of chronic kidney disease and at initiation of dialysis treatment. Health Qual Life Outcomes. 2012;10:71.

32. Pedone C, Corsonello A, Bandinelli S, Pizzarelli F, Ferrucci L, Incalzi RA. Relationship between renal function and functional decline: role of the estimating equation. J Am Med Dir Assoc. 2012;13:84.e11-4.

33. Workeneh BT, Mitch WE. Review of muscle wasting associated with chronic kidney disease. Am J Clin Nutr. 2010;91:1128S-32S.

34. Devrome AN, Maclntosh BR. Force-velocity relationship during isometric and isotonic fatiguing contractions. J Appl Physiol. 2018;125: $706-14$.

35. Fois A, Chatrenet A, Cataldo E, Lippi F, Kaniassi A, Vigreux J, et al. Moderate protein restriction in advanced CKD: a feasible option in an elderly, HighComorbidity Population A Stepwise Multiple-Choice System Approach. Nutrients. 2019:11:36.

36. Ramírez-Vélez R, Correa-Bautista JE, García-Hermoso A, Cano CA, Izquierdo M. Reference values for handgrip strength and their association with intrinsic capacity domains among older adults. J Cachexia Sarcopenia Muscle. 2019:10:278-86.

37. Veni T, Boyas S, Beaune B, Bourgeois H, Rahmani A, Landry S, et al. Handgrip fatiquing exercise can provide objective assessment of cancer-related fatigue: a pilot study. Support Care Cancer. 2018;27:229-38.

38. Poole DC, Burnley M, Vanhatalo A, Rossiter HB, Jones AM. Critical power: an important fatigue threshold in exercise physiology. Med Sci Sports Exerc. 2016:48:2320-34.

39. Kwakkenbos L, Willems LM, Baron M, Hudson M, Cella D, van den Ende $\mathrm{CHM}$, et al. The comparability of English, French and Dutch scores on the functional assessment of chronic illness therapy-fatigue (FACIT-F): an assessment of differential item functioning in patients with systemic sclerosis. PLoS One. 2014;9:e91979.

40. Gentile S, Delarozière JC, Favre F, Sambuc R, San Marco JL. Validation of the French "multidimensional fatigue inventory" (MFI 20). Eur J Cancer Care (Engl). 2003;12:58-64.

41. Charlson ME, Pompei P, Ales KL, MacKenzie CR. A new method of classifying prognostic comorbidity in longitudinal studies: development and validation. J Chronic Dis. 1987:40:373-83.

42. Borges MCC, Vogt BP, Martin LC, Caramori JCT. Malnutrition inflammation score cut-off predicting mortality in maintenance hemodialysis patients. Clin Nutr ESPEN. 2017;17:63-7.

43. May SE, Keir PJ. Effect of wrist posture, rate of force development/relaxation, and isotonic contractions on finger force independence. J Electromyogr Kinesiol. 2018:38:215-23.

44. Sanei K, Keir PJ. Independence and control of the fingers depend on direction and contraction mode. Hum Mov Sci. 2013;32:457-71.

45. Dai C, Hu X. Extracting and classifying spatial muscle activation patterns in forearm flexor muscles using high-density electromyogram recordings. Int J Neural Syst. 2019;29:1850025.

46. Opplert J, Babault N. Acute effects of dynamic stretching on muscle flexibility and performance: an analysis of the current literature. Sports Med. 2018:48:299-325.

47. Simic L, Sarabon N, Markovic G. Does pre-exercise static stretching inhibit maximal muscular performance? A meta-analytical review. Scand J Med Sci Sports. 2013;23:131-48.

48. Maffiuletti NA, Aagaard P, Blazevich AJ, Folland J, Tillin N, Duchateau J. Rate of force development: physiological and methodological considerations. Eur J Appl Physiol. 2016;116:1091-116.

49. De Luca CJ. The use of surface electromyography in biomechanics. J Appl Biomech. 1997;13:135-63.

50. Konrad P. A Practical Introduction to Kinesiological Electromyography; 2006. p. 61. 
51. Burnley M, Vanhatalo A, Jones AM. Distinct profiles of neuromuscular fatigue during muscle contractions below and above the critical torque in humans. J Appl Physiol. 2012;113:215-23.

52. Lin M-Y, Chiu Y-W, Lee C-H, Yu H-Y, Chen H-C, Wu M-T, et al. Factors associated with CKD in the elderly and nonelderly population. Clin J Am Soc Nephrol. 2013;8:33-40.

53. Almurdhi MM, Reeves ND, Bowling FL, Boulton AJM, Jeziorska M, Malik RA. Reduced lower-limb muscle strength and volume in patients with type 2 diabetes in relation to neuropathy, intramuscular fat, and vitamin D levels. Diabetes Care. 2016;39:441-7.

54. Orlando G, Balducci S, Bazzucchi I, Pugliese G, Sacchetti M. Neuromuscular dysfunction in type 2 diabetes: underlying mechanisms and effect of resistance training. Diabetes Metab Res Rev. 2016;32:40-50.

55. Giglio BM, Mota JF, Wall BT, Pimentel GD. Low handgrip strength is not associated with type 2 diabetes mellitus and hyperglycemia: a populationbased study. Clin Nutr Res. 2018;7:112-6.

\section{Publisher's Note}

Springer Nature remains neutral with regard to jurisdictional claims in published maps and institutional affiliations.

Ready to submit your research? Choose BMC and benefit from:

- fast, convenient online submission

- thorough peer review by experienced researchers in your field

- rapid publication on acceptance

- support for research data, including large and complex data types

- gold Open Access which fosters wider collaboration and increased citations

- maximum visibility for your research: over $100 \mathrm{M}$ website views per year

At BMC, research is always in progress.

Learn more biomedcentral.com/submissions 